\title{
Measurement of aerosol properties during wintertime in Beijing
}

Misti Levy Zamora, ${ }^{1,3}$, Jianfei Peng ${ }^{1,2, \dagger}$, Min $\mathrm{Hu}^{2, *}$, Song Guo,2, Wilmarie Marrero-Ortiz ${ }^{1}$, Dongjie Shang ${ }^{2}$, Jing Zheng ${ }^{2}$, Zhuofei $\mathrm{Du}^{2}$, Zhijun $\mathrm{Wu}^{2}$ \& Renyi Zhang,2,*

${ }^{1}$ Department of Atmospheric Sciences and Department of Chemistry, Center for Atmospheric Chemistry and the Environment, 5 Texas A\&M University, College Station, TX 77843, USA

${ }^{2}$ State Key Joint Laboratory of Environmental Simulation and Pollution Control, College of Environmental Sciences and Engineering, Peking University, Beijing 100871, China

${ }^{3}$ Department of Environmental Health and Engineering, Johns Hopkins Bloomberg School of Public Health, 615 N. Wolfe St., Baltimore, Maryland 21205, USA

${ }^{\dagger}$ These authors contribute to this paper equally.

*Correspondence to: Min Hu (minhu@pku.edu.cn); Renyi Zhang (Renyi-Zhang@tamu.edu) 


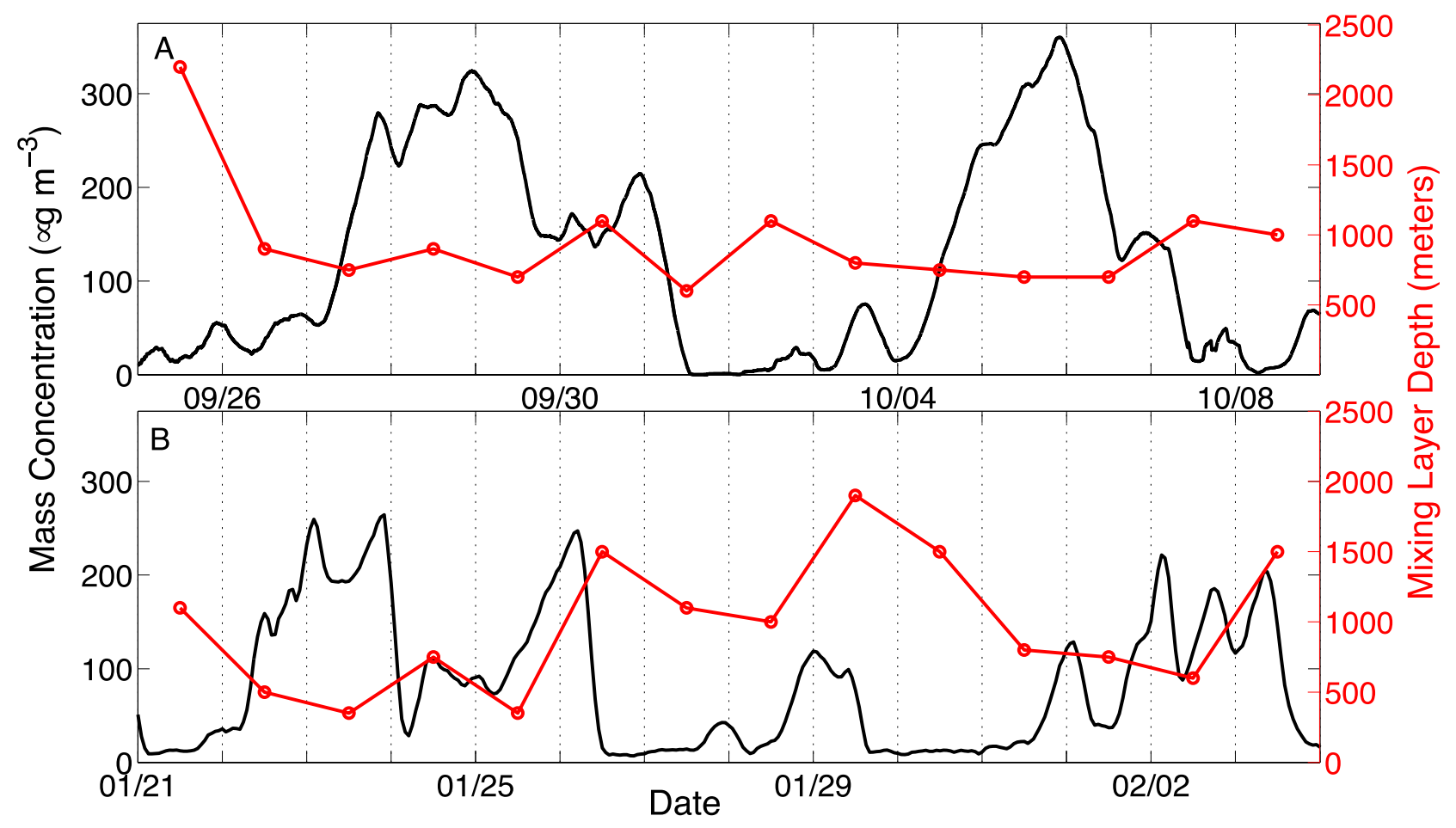

Figure S1. A comparison of the mixing layer depth (m) determined by the HYSPLIT model and the mass concentration ( $\mu \mathrm{g}$ $\mathrm{m}^{-3}$ ) in (A) Autumn 2013 and (B) Winter 2015. 